\title{
Supporting real-time distributed computer-controlled systems with multi-hop P-NET networks
}

\author{
Eduardo Tovara, Francisco Vasquesb, Alan Burnsc
}

\begin{abstract}
Fieldbus communication networks aim to interconnect sensors, actuators and controllers within process control applications. Therefore, they constitute the foundation upon which real-time distributed computer-controlled systems can be implemented. P-NET is a "eldbus communication standard, which uses a virtual token-passing medium-access-control mechanism. In this paper pre-runtime schedulability conditions for supporting real-time tra \$c with P-NET networks are established. Essentially, formulae to evaluate the upper bound of the end-to-end communication delay in P-NET messages are provided. Using this upper bound, a feasibility test is then provided to check the timing requirements for accessing remote process variables. This paper also shows how P-NET network segmentation can signi"cantly reduce the end-to-end communication delays for messages with stringent timing requirements.
\end{abstract}

\section{Keywords}

Distributed computer-controlled systems; P-NET "eldbus networks; Real-time communications; Message response time analysis

\section{Introduction}

Local area networks (LANs) are becoming increasingly popular in industrial computer-controlled systems. LANs allow "eld devices like sensors, actuators and controllers to be interconnected at low cost, using less wiring and requiring less maintenance than point-topoint connections (Lenhart, 1993). Besides the economic aspects, the use of LANs is also reinforced by the increasing decentralisation of control and measurement tasks, as well as by the increased use of intelligent microprocessor-controlled devices.

Broadcast LANs aimed at the interconnection of sensors, actuators and controllers are commonly known as "eldbus networks. In the past, the scope of "eldbuses was dominated by vendor-speci"c solutions, which were mostly restricted to speci"c application areas. Moreover, the concepts behind each proposed network were highly dependent on the manufacturer of the automation system. Each one had di!erent technical implementations and claimed to ful"1 di!erent application requirements, or to ful"1 the same requirements but with di!erent technical solutions (Cardoso \& Tovar, 1996). More recently, vendor-independent standardised "eldbuses, supporting the open system concept, have started to be commonly used. Particular relevance must be given to the European Standard EN 50170 (Cenelec, 1996), which encompasses three widely used "eldbuses: P-NET (Pnet, 1994), PROFIBUS (Pro"bus, 1992) and FIP (Afnor, 1990).

This paper addresses the ability of P-NET to cope with the real-time requirements of distributed computer-controlled systems (DCCS). In essence, timing requirements mean that messages must be sent and received within a bounded interval, otherwise a timing fault is said to occur. That means, for instance, that a control device must be able to read data from a remote sensor within a speci"ed interval, whatever the network load.

The P-NET medium-access-control (MAC) protocol is based on a virtual token-passing procedure, used by master stations to grant the bus access to each one of 
them, and a master slave procedure used by master stations to communicate with slave stations. This master slave interaction is called a message cycle: the master sends a request frame and the addressed slave immediately sends a response frame. At each of the visits of the token, a master is able to perform, at most, one message cycle.

Typically, the process-relevant devices (sensors and actuators) are accessed through a slave network-interface, whereas the distributed control algorithms reside at master stations. Therefore, in P-NET, the end-to-end communication delay (Tindell, Burns \& Wellings, 1995) for master slave transactions (those that typically deal with real-time tra $\$ c$ ) is composed of the following four major components:

1. generation delay: the time elapsed between the release of the sender task and the queuing of the related message request;

2. queuing delay: the time taken by a message request to access the communication medium after being queued;

3. transmission delay: the time taken by a message request to be transmitted on the communication medium and processed at the slave side, added to the time taken by the message response to be transmitted back to the master;

4. delivery delay: the time taken by the master's application task to process a message response.

The queuing delay is a consequence of the contention not only between message requests from the same master but also with message requests from other masters. The

impact of the "rst factor in the overall queuing delay

depends on the policy used to queue the message requests, while the second factor depends on the behaviour of the token-passing procedure. Therefore, an evaluation of the worst-case queuing delay of message requests is of

paramount importance to guarantee the messages' timing requirements. The end-to-end communication delay is referred to in this paper as the message response time.

The remainder of this paper is organised as follows. Section 2 describes the most important concepts behind

P-NET networks. Particular relevance is given to the behaviour and timing characteristics of its MAC protocol. Based on these characteristics, an upper bound for the message response time is derived in Section 3. This upper bound gives a su\$cient pre-run-time schedulability condition; that is, if the messages' relative deadlines are greater than their worst-case response time, then the real-time requirements of the supported application are guaranteed. As it will be shown, the upper bound for the message response time is a function of the number of masters in the network segment. This motivates the analysis performed in Section 4, which addresses the response time analysis for messages relayed through P-NET hop- ping devices. The idea behind this analysis is the following one. In P-NET networks several bus segments can be interconnected into a larger network using hopping devices, in such a way that any master on the network can transparently access any node within the network. Such a segmentation has e!ective advantages in terms of the real-time characteristics of the overall network. As each segment has independent virtual token-passing procedures, the token rotation time decreases. If nodes with more stringent message transactions are placed in the same network segment, the response time for those messages will be smaller than in the single-segment approach. Nonetheless, transactions that are relayed through one or more hopping devices may be required. Such multihop transactions will su!er an increase in their response times. In Section 4 an upper bound for the multi-hop message response time is derived. Both the basic and the multi-hop response time analysis assume worst-case peak-load situations. This allows the determination of su $\$$ cient but not necessary pre-run-time schedulability conditions. In the appendix, the level of pessimism in these pre-run-time schedulability conditions is investigated. In Section 5 a numerical example is presented to show how to apply the pre-run-time schedulability conditions. With this example, the advantages and criteria for segmenting a P-NET network are presented, and some conclusions are drawn.

\section{A brief description of P-NET}

P-NET (process network) was designed as a communications link between distributed process-control sensors, actuators and small programmable controllers. It has recently gained an increased role, as it became (along with PROFIBUS and FIP) a European Standard, the EN 50170$\}$ General-Purpose Field Bus Communication System.

P-NET is a multi-master standard based on a virtual token-passing (VTP) scheme. In P-NET all communication is based on a message transaction principle, where a master sends a request and the addressed slave immediately returns a response. Fig. 1 illustrates the hybridoperating mode of the P-NET's MAC.

The P-NET standard uses a data rate of $76800 \mathrm{bps}$. This data rate resulted from weighing up the con\#icting requirement for data to be transported as fast as possible, but not at such speed as to negate the use of standard microprocessor UARTS, or restrict the usable distance or cable type (Jenkins, 1997).

The VTP scheme is implemented using two protocol counters. The "rst one, the access counter (AC), holds the node address of the master currently transmitting. When a request has been completed and the bus has been idle for 40 bit periods $(520 \mu \mathrm{s}$ at $76.8 \mathrm{Kbps})$, each one of the access counters is incremented by one. The master whose 
access counter value equals its own unique node address is said to be holding the token, and is allowed to access the bus. When, as the access counter is incremented, it exceeds the 'maximum number of mastersa, the access counter in each master is reset to one. This allows the "rst master in the cycling chain to gain access again.

The second counter, the idle bus bit period counter (IBBPC), increments for each inactive bus bit period. Should any transactions occur, the counter is reset to zero. As explained above, when the bus has been idle for 40 bit periods following a transfer, all the access counters are incremented by one, and the next master is thus allowed to access the bus.

If a master has nothing to transmit (or indeed is not even present), the bus will continue to be inactive. Following a further period of $130 \mu \mathrm{s}$ (10 bit periods), the idle bus bit period counter will have reached $50(60,70,2)$ so all the access counters will be incremented again, allowing the next master access. The virtual token-passing will continue every $130 \mu \mathrm{s}$, until a master does require access.

The P-NET standard permits each master to perform only one message transaction (later de"ned as message

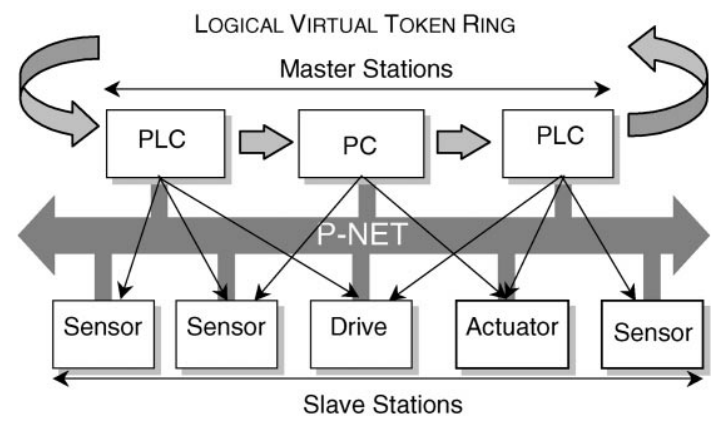

Fig. 1. Token passing and master\}slave procedures in P-NET networks. cycle) per token visit. This is an important idea for the remainder of this paper.

After receiving the token, the master must transmit a request before a certain time has elapsed. This is denoted as the master's reaction time, and the standard imposes a worst-case value of up to 7 bit periods. A slave is allowed to access the bus, between 11 and 30 bit periods after receiving a request, measured from the beginning of the stop bit in the last byte of the frame. The maximum allowed delay is then $390 \mu$ s (corresponding to 30 bit periods). Later on, this delay will also be denoted as the slave's turnaround time. For a better understanding of the basic MAC procedures and the notation used, refer to Fig. 2.

Before proceeding with the worst-case response time analysis, it is important to understand the idea of a PNET message cycle length. A P-NET frame contains "ve "elds: node address "eld ( 2 bytes); control/status "eld (1 byte); information length "eld ( 1 byte); information "eld (0\}63 bytes); error detection "eld (1\}2 bytes). The node address "eld may have up to 24 frame bytes. P-NET uses these complex addresses if multiple segments are used and special devices (P-NET hopping devices) are used to relay frames between the di!erent segments.

As each frame byte in P-NET actually corresponds to 11 bits, a frame may have up to 759 bits $(69 \times 11$ bits). In P-NET all the frame bytes are sent asynchronously, with one start bit (logical zero), 8 data bits (with LSB "rst), one address/data bit and one stop bit. Within a frame, a start bit must immediately follow a stop bit.

Thus, considering the case that both the request and response frames have 759 bits (realistically it is more likely that either the request will be longer, in cases of data being written to a slave, or the response will be longer, in cases of data being received from a slave), the overall sum for the longest message cycle is 1548 bit periods, corresponding to $20.15 \mathrm{~ms}$ at $76800 \mathrm{bps}$. This

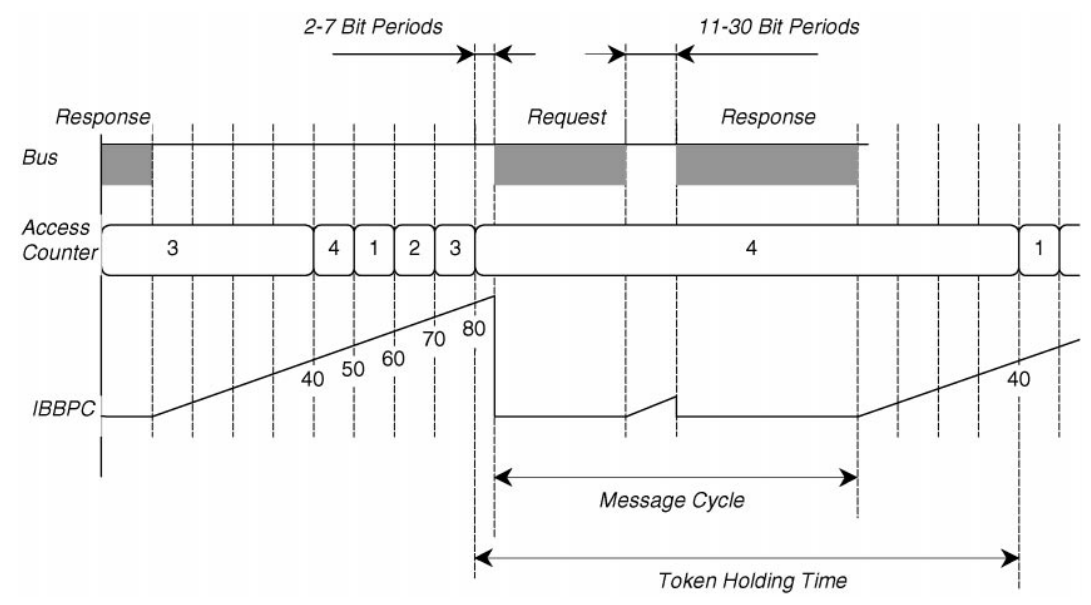

Fig. 2. An example of virtual token-passing in P-NET networks. 
includes the worst-case slaves' turnaround time (30 bit periods).

\section{A basic pre-run-time schedulability analysis}

The following pre-run-time schedulability analysis provides su\$cient conditions to guarantee the timing requirements of P-NET messages. Some of the results presented in this section were introduced in Tovar and

Vasques (1998a,b).

\subsection{Network and message models}

Consider a network with $n$ masters, with addresses ranging from 1 to $n$. Each master accesses the network according to the VTP scheme; hence, "rst master 1, then masters 2, 3,2 until master $n$, and then again masters 1 , 2,2 . Slaves will have network addresses higher than $n$.

The following message stream model is assumed:

$$
S_{i}^{k}=\left(C_{i}^{k}, D_{i}^{k}\right)
$$

$S k$ de"nes a message stream $i$ in master $k(k \cdots 1, \mathbf{Z}, n)$. A message stream is a temporal sequence of message cycles concerning, for instance, the remote reading of a speci"c process variable. $C k$ is the longest message cycle dufatignge of ftream $S k$. $D k$ is the relative deadline of stream $S k$ that is, the maximum admis-

sible end-to-end communication delay for that message cycle.

\subsection{Upper bound for the virtual token cycle}

In P-NET each master is allowed to perform, at most, one message cycle per token visit. Therefore, the evaluation of the P-NET token cycle time (maximum time between two consecutive token arrivals at a master) is of paramount importance for the estimation of the end-toend communication delay. It is obvious that an upper bound for the P-NET token cycle time (denoted as $<$ ) equals the sum of the maximum token-holding time in every master, and its value is

$$
V=\sum_{i=1}^{n}\left(7 \times b p+\max _{j-1 \ldots n s^{i}}\left(C_{j}^{i}\right)+40 \times b p\right),
$$

where $7 \times b p$ represents the longest master's reaction time and $40 \times b p$ represents the token-passing time after performing a message cycle. The longest message cycle in a master $l, \max _{j=1,2, n s} l(C)$, includes the time needed to transmit both the request and response frames, the slave's turnaround time (which in P-NET is bounded to $30 \times b p$ ) and propagation delays.

\subsection{A basic pre-run-time schedulability condition}

In P-NET, the message requests are queued in a "rstcome-"rst-served (FCFS) queue. The maximum number of pending requests is $n s k$, since if there were two pending message requests from the same $S k$, this would mean that a deadline had been missed. The peak-load condition occurs when $n s k$ requests are simultaneously made just after the token passes to the next master. In this case, the

transmission of the last of those nsk requests is deferred by $n s k$ visits of the token. If the last message is the one with the most stringent deadline, a `priority inversiona with the length (nsk $\mathbf{I} 1) \times<$ occurs.

If $R$ denotes the upper bound for the message response time of stream $S k$, this upper bound is given by

$$
R_{i}^{k}=n s^{k} \times V+7 \times b p+C_{i}^{k}+\lambda_{i}^{k},
$$

where $A k$ aggregates both the upper bounds for the generation and the delivery delays for $S k$ (Fig. 3).

It is now possible to introduce the pre-run-time schedulability condition, which is a su\$cient condition to guarantee that real-time messages are transmitted before their deadlines. In P-NET, a set of message streams is schedulable if, at each master $k$ :

$$
D_{i}^{k} \geqslant R_{i}^{k}, \quad \forall_{i \in\left\{1, \ldots, n^{k}\right\}} \text {. }
$$

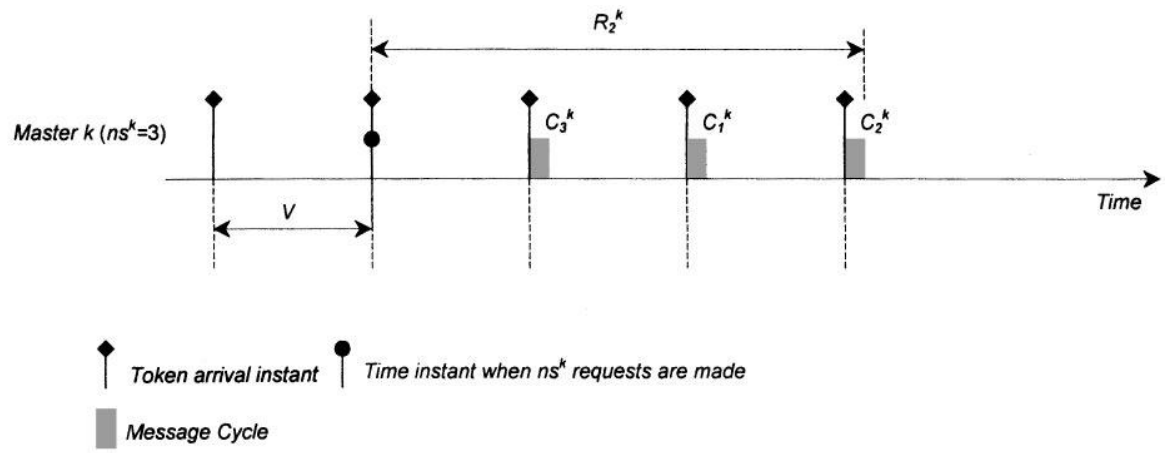

Fig. 3. An example of a message response time evaluation. 


\section{Schedulability analysis for multi-hop P-NET networks}

Hopping P-NET devices (labelled as gateways in the standard, but termed hopping devices in this paper) allow the interconnection of di!erent network segments, each one with independent logical virtual token-passing schemes. In P-NET, the function of a gateway is to isolate two or more bus segments, and to automatically route a frame between the connected buses. In the authors opinion, and according to ISO-OSI de"nitions, the P-NET gateways combine techniques used in bridges and routers, and thus the term 'hopping devicesa is preferred.

The P-NET multi-segment feature allows for routing
through up to ten hopprng devices. These mult-hoppirg capabilities are based on simple rules for address conversion inside the hopping devices.

P-NET supports four types of addresses: simple, complex, extended and response address types. The simple and response address types use only 2 bytes (destination and source addresses). The extended address uses 4 bytes ( 2 destination and 2 source address bytes) and the complex address may use up to 24 bytes. P-NET uses the complex addressing scheme to route frames through hopping devices. This complex address explicitly addresses each intermediate device.

In P-NET, hopping devices isolate tra $\$ \mathrm{c}$ between $\mathrm{P}$ NET segments. If the di!erent segments group interrelated masters and slaves, the overall real-time capabilities are improved, as the virtual token cycle time in each single segment becomes smaller. However, if a particular stream relates a master and a slave in two distinct segments, that stream will have a higher response time. In this section an analysis for deriving the upper bound of the response time for messages that are to be relayed

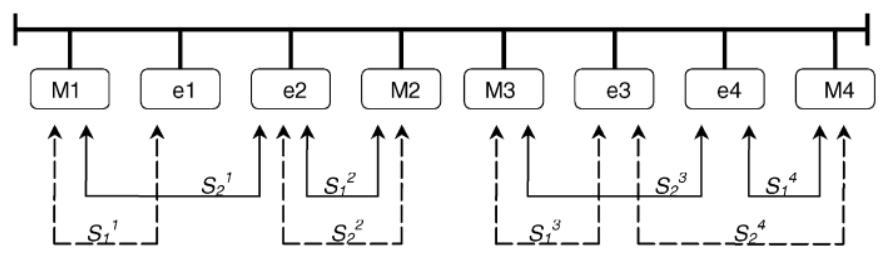

Fig. 4. A P-NET network example. through a number of P-NET hopping devices is provided.

\subsection{Motivation}

Suppose a P-NET network composed of four masters (M1, M2, M3, M4) and four slaves (e1, e2, e3, e4), all connected to the same network segment. Each one of the masters deals with two message streams, as shown in Fig. 4.

Considering that the maximum token holding time in each station is $250 \times b p$ (this means that the longest. message cycles of all the masters are equal: $C^{\prime}$.

$\left.203 \times b p, \forall_{, k}\right)$, then the pre-run-time schedulability condition (4) is (ignoring A): DS $[2 \times(4 \times(250) \not \boldsymbol{H}$ $(7 \not \# 203)] \times b p \boldsymbol{C}^{2} 2210 \times 1 / 76800 \cdots 28.8 \mathrm{~ms}$.

The example in Fig. 4 illustrates, on a reduced scale, the advantages of segmentation. In fact, the whole network could be composed of two segments, grouping M1, $\mathrm{M} 2$, e1 and e2 in one segment, and M3, M4, e3 and e4 in another segment (Fig. 5). For simpli"cation, any of the existing masters (M1, M2, M3 or M4) is used to implement the multi-hopping features.

As none of the message streams are to be relayed through the hopping device, the pre-run-time schedulability condition becomes: $D S[2 \times(3 \times 250) \not=210] \times$ $b p{ }^{\bullet} 1710 \times 1 / 76800 \cdots 22.3 \mathrm{~ms}$.

However, in more complex systems, it is unlikely that all the message streams can be restricted to their own segments. As real implementations of slave nodes group several I/O points in racks, it is possible that speci"c information \#ows will demand inter-operation between masters and slaves in di!erent network segments.

\subsection{Sequence of transactions in multi-hop message streams}

Apart from having a longer address "eld, multi-hop message streams (message streams that are relayed through at least one hopping device) will have additional queuing and access delays.

Fig. 6 illustrates the message sequence corresponding to master/slave transactions through two hopping devices. It is important to notice that each hopping device

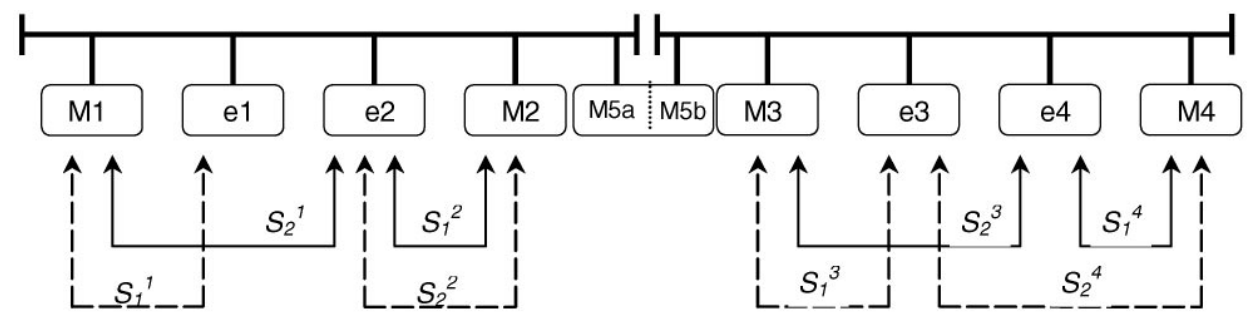

Fig. 5. Using one hopping device. 


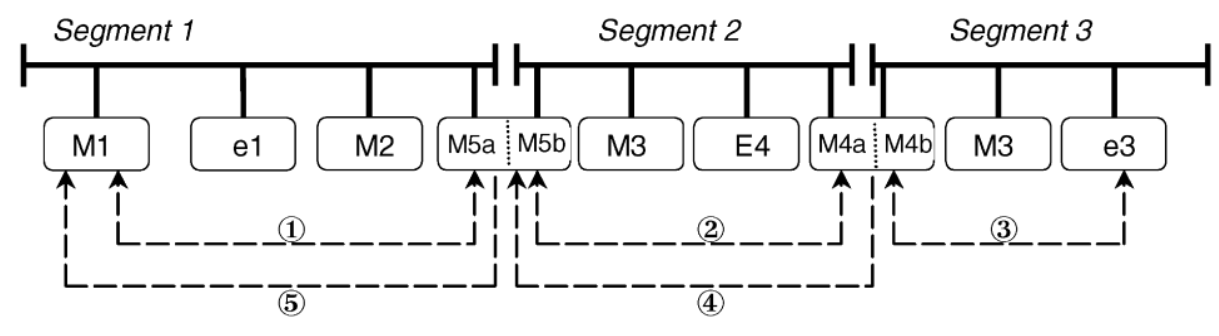

Fig. 6. An example of a multi-hop transaction.

embodies two masters (and in the general case, as many masters as the number of segments that it interconnects).

If master M1 (in network segment 1) requires the reading of a sensor associated with slave $\mathrm{e} 3$ (in network segment 3), hopping devices M5 and M4 are used to relay the message stream. The sequence of message transactions is as follows.

1. When M1 gains access to the network (segment 1), and the message is the "rst one in the outgoing queue, M1 sends a request and M5a responds immediately with an 'answer due latera.

2. When M5b gains access to segment 2, and the message is the "rst one in the outgoing queue, M5b sends the request and M4a responds immediately with an 'answer due latera.

3. When M4b gains access to segment 3 , and the message is the "rst one in the outgoing queue, M4b sends the

request and slave e 3 responds immediately with the requested information.

4. When M4a gains access to segment 2, and the message containing the required information is the "rst one in the outgoing queue, M4a sends a request without a response to M5b.

5. When M5a gains access to segment 1, and the message is the "rst one in the outgoing queue, M5a sends a request (containing the required information) without a response to $\mathrm{M} 1$.

So, in general, if $h$ represents the number of intermediate hopping devices through which a message stream is to be relayed, there will be $2 \times \boldsymbol{h} \not \boldsymbol{1} 1$ queueing and access delays to be considered.

\subsection{Pre-run-time schedulability condition for multi-hop message streams}

Each P-NET segment has its own virtual token-rotation procedure. Thus, the maximum virtual token cycle time in a segment $\mathrm{can}$ be de"ned as

$V_{\xi}=\sum_{i \in \xi}\left(7 \times b p+\max _{j-1 \ldots n s^{\prime}}\left(C_{j}^{j}\right)+40 \times b p\right)$.
The upper bound for the response time of a message from stream $S k$ can be derived as follows. If the message stream is to be relayed through 0 hopping devices, then $R k$ is

$$
R_{i}^{k}=n s^{k} \times V(k)+7 \times b p+C_{i}^{k}+\lambda_{i}^{k},
$$

where $<(k)$ corresponds to the upper bound for the virtual token rotation time of the network segment to which station $k$ belongs.

If the message stream is to be relayed through 1 hopping device, then $R k$ is:

$$
\begin{aligned}
R_{i}^{k}= & \left(n s^{k}+n s^{r_{1}}\right) \times V(k)+n s^{r_{2}} \times V\left(r_{2}\right) \\
& +\sum_{j=1}^{3}\left(7 \times b p+C_{j}+\lambda_{j}\right)+2 \times \phi,
\end{aligned}
$$

where $r 1$ is the hopping master in the same segment as master $k$, and $r_{2}$ is the hopping master in the other

segment. The summation represents the length of each of the $2 \times h \neq \# 1$ message transactions that will occur (using a simpli"ed notation $C_{j}$ ) and the respective protocol overheads (using a simpli"ed notation $A_{j}$ ). The symbol $\phi$ stands for the time needed by the hopping device to transfer frames between communication stacks.

If the message stream is to be relayed through $h$ hopping devices (with $h \mathbf{5}$ 2), then $R \mathrm{k}$ is

$$
\begin{aligned}
R_{i}^{k}= & \left(n s^{k}+n s^{r^{n}}\right) \times V(k) \\
& +\sum_{j=1}^{h-1}\left[\left(n s^{r_{2 \times j}}+n s^{r_{2 \times j+1}}\right) \times V\left(r_{2 \times j}\right)\right] \\
& +n s^{r_{2 \times} \times} \times V\left(r_{2 \times h}\right) \\
& +\sum_{j=1}^{2 \times h+1}\left(7 \times b p+C_{j}+\lambda_{j}\right)+2 \times h \times \phi,
\end{aligned}
$$

where $r_{1}$ is the hopping device master in the same segment as master $k$ and $r_{2}, r_{3}, \mathbf{2}, r_{2 \times h}$ are the hopping device masters which relay the message from master $k$ to its destination. For example, for the scenario shown in

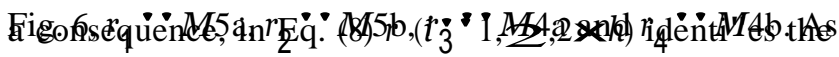
masters in hopping devices according to the physical sequence from master $k$ to the addressed slave devices 
Number of message streams related to each master

\begin{tabular}{llllllll}
\hline Master & 1 & 2 & 3 & 4 & 5 & 6 & 7 \\
\hline nsk & 3 & 4 & 3 & 2 & 1 & 4 & 5 \\
$\left.\max C k_{j}\right\}$ & $200 b p$ & $200 b p$ & $200 b p$ & $200 b p$ & $200 b p$ & $200 p b$ & $200 b p$ \\
\hline
\end{tabular}

and not according to the sequence of transactions (see Section 4.3).

As for the non-segmented case (Eqs. (3) or (6)), the same su\$cient P-NET pre-run-time schedulability condition (inequality (4) $\ D k 5 R k$ ) can be used to guarantee that real-time multi-hop message streams are processed before their deadlines. Depending on the number of hopping devices a message is relayed through, Eqs. (6), (7) or (8) is used to evaluate the upper bound of the message response time.

\section{Numerical results}

In the previous section, a su\$cient pre-run-time schedulability condition for supporting real-time communications with P-NET has been provided. It was also demonstrated that the message response time can become much smaller if the network nodes are distributed by a number of P-NET network segments, each one grouping as many inter-related network nodes as possible. In this way tighter message deadlines can then be supported.

In this section, a numerical example is provided, which exempli"es what a user of P-NET can obtain from the proposed pre-run-time schedulability conditions. Al-

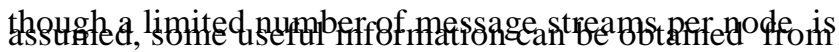

this example:

1. how the maximum upper bound of each messagestream's response time, in both a non-segmented and segmented P-NET network can be evaluated;

2. how a P-NET network can be segmented in order to reduce the maximum upper bound for the message response time.

In this speci"c example, a comparison is also made between the response time's upper bound in a non-segmented and a segmented P-NET network, clearly showing the impact of network segmentation.

Assume that a distributed computer-controlled system must be implemented using eight master networks. Also assume that all message cycle lengths are bounded to $200 \times b p(2.6 \mathrm{~ms}$ at $76800 \mathrm{bps})$. The number of streams related to each master is shown in Table 1 (a total of 28 message streams, distributed by eight masters).
Table 2

Upper bound for the message response times

\begin{tabular}{lllllllll}
\hline Master & 1 & 2 & 3 & 4 & 5 & 6 & 7 & 8 \\
\hline$R k(\mathrm{~ms})$ & 79.8 & 105.5 & 79.8 & 54.1 & 28.4 & 105.2 & 131.2 & 156.9 \\
\hline
\end{tabular}

In this case, the upper bound for the virtual token cycle is $>8 \times 247 b p=25.7 \mathrm{~ms}$. Therefore, using Eq. (3), the upper bound for the message response times is as shown in Table 2 (all streams in the same master will have the same upper bound for their response times). Note that the generation and delivery delays at the application process level are ignored, and must be evaluated at the level of the application process software. However, ignoring such delays is not of major importance, and as PNET operates at 76800 bps they will be usually much smaller than the transmission and queuing delays.

If, for example, the application imposes deadlines smaller than $105.5 \mathrm{~ms}$ for the message streams of master 2 , or less than $28.4 \mathrm{~ms}$ for the message streams of master 5 , then the message stream set would not be schedulable.

Suppose that by re-organising the network into three network segments, as shown in Fig. 7, only two message streams are multi-hop streams: $S 11$ and $S \mathbf{8}$. Then, tighter

\section{all but those}

streams.

Assume that streams $S 1_{1}$ and $S 8_{2}$ correspond to remote accesses, to slaves in segment 2 and segment 8 , respectively. Table 3 illustrates the routing sequence for those streams.

These two streams will impose two additional message streams (resulting from messages being relayed through this hopping device) in masters 3 and 4, and one additional message stream in masters 6 and 7 . Table 4 re\#ects the aggregate number of message streams per master station.

If, for simpli"cation, $\phi$ and A components from Eqs. (7) and (8) are ignored, as well as the additional byte addresses in the multi-hop streams (thus maintaining $200 \times b p$ as the value for the longest message cycle in each master), then the results, shown in Table 5, are obtained.

By implementing the proposed network segmentation, an important reduction of worst-case response times can be achieved, as illustrated in Tables 6 and 7 . 


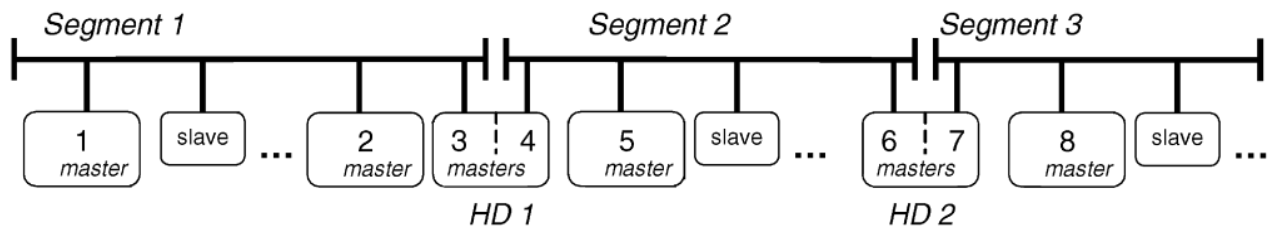

Fig. 7. Proposed segmentation of the network.

Table 3

Routing sequence (master IDs) for the multi-hop streams

\begin{tabular}{llllll}
\hline & $h$ & $r_{1}$ & $r_{2}$ & $r_{3}$ & $r_{4}$ \\
\hline$S 1$ & 1 & 3 & 4 & $*$ & $*$ \\
$S 8$ & 2 & 7 & 6 & 4 & 3 \\
\hline
\end{tabular}

Table 4

Aggregate number of message streams related to each master

\begin{tabular}{lllllllll}
\hline Master & 1 & 2 & 3 & 4 & 5 & 6 & 7 & 8 \\
\hline$n s k$ & 3 & 4 & 5 & 4 & 1 & 5 & 6 & 6 \\
\hline
\end{tabular}

Table 5

Upper bound for the virtual token Cycle in each segment (Eq. (5))

\begin{tabular}{llll}
\hline Segment & 1 & 2 & 3 \\
\hline$<(\mathrm{ms})$ & 9.65 & 9.65 & 6.43 \\
\hline
\end{tabular}

Obviously, for the multi-hop message streams $S 1_{1}$ and $S \&$ the upper bounds for their response times increase as compared to the "gures given in Table $2(79.8 \mathrm{~ms}$ and $156.9 \mathrm{~ms}$, respectively).

The values are as follows. For $R 1$ Eq. (7) is used and Table 4 gives the number of streams for the masters. Its value is $R 1 \cdots(n s 1 \boldsymbol{\#} n s 3) \mathbf{x}<(1) \not{\#} n s 4 \mathbf{x}<(4) \boldsymbol{\#}$ $3 \times 207 b p \cdots(3 \not \# 5) \times 9.65 \not \# 4 \times 9.65 \not \# 8.08 \cdots 123.88 \mathrm{~ms}$.

For $R 8$ Eq. (8) is used and Table 4 gives the number of streams for the masters. Its value is $R_{8} \cdots$ $(n s 8 \not \# n s 7) \times<(8) \not \#(n s 6 \not \# n s 4) \times<<(6) \not \# n s 3 \mathbf{x}<43)$

$\not \# 5 \times 207 b p \cdots 12 \times 6.43 \not \# 9 \times 9.65 \not \# 5 \times 9.65 \not 13.47$ - $225.73 \mathrm{~ms}$.

The upper bound for the response time of message stream $S_{1}$ (for which $h{ }^{\cdots}$ ) becomes $155 \%$ higher (related to the value given by Table 2), whereas message stream $S_{2}^{8}$ (for which $h \cdot 2$ ) becomes $144 \%$ higher.

The impact of network segmentation on the response time of both single-segment and multi-hop message streams is therefore clear.
Table 6

Upper bound for the response times (single-segment streams Eq. (6))

\begin{tabular}{lllllllll}
\hline Master & 1 & 2 & 3 & 4 & 5 & 6 & 7 & 8 \\
\hline RHk (ms) & 31.65 & 41.3 & 50.95 & 41.3 & 12.35 & 50.95 & 41.28 & 41.28 \\
\hline
\end{tabular}

Table 7

Percentage of the upper bound obtained as compared to Table 2

\begin{tabular}{lllllllll}
\hline Master & 1 & 2 & 3 & 4 & 5 & 6 & 7 & 8 \\
\hline$R H k / R k$ & $39.6 \%$ & $39.1 \%$ & $63.8 \%$ & $76.3 \%$ & $43.5 \%$ & $48.4 \%$ & $31.4 \%$ & $36.3 \%$
\end{tabular}

\section{Conclusions}

In this paper, schedulability conditions for supporting real-time distributed computer-controlled systems with P-NET networks are provided. Both non-segmented and segmented P-NET networks are analysed.

This paper shows the advantages of using P-NET hopping devices for supporting network segmentation, since a signi"cant reduction in response times can be achieved in most message transactions. However, the system designer mustclearly understand that such reductions are not possible for inter-segment message transactions. Therefore, care should be taken to group masters and slaves involved in message transactions with stringent deadlines in the same network segment.

The proposed schedulability analysis provides su\$cient conditions to guarantee message timing requirements. As these schedulability conditions are derived under peak-load assumptions, they present a certain level of pessimism. Therefore, an investigation of this pessimism is provided in the Appendix. This may allow system engineers to design P-NET network-based DCCS, within which some message transactions have softer real-time requirements.

\section{Acknowledgements}

The authors acknowledge the useful comments made by the anonymous reviewers. This work was partially 
supported by ISEP (project REMETER), by FLAD (project SISTER 471/97) and by DEMEGI/FEUP.

\section{Appendix A}

The pre-run-time schedulability conditions derived in this paper are su $\$ c i e n t$ but not necessary. This is a result of trying to develop an approach to guaranteed timeliness based on the worst-case peak-load conditions. It is therefore pertinent to present an evaluation of the pessimism that exists in the pre-run-time schedulability conditions. Revisiting Eq. (3), used in the case of nonsegmented networks, the following sources of pessimism are present:

(1) In the priority inversion factor $n s k$ :

(a) it is a worst-case scenario that all messages would be requested to be transferred in the period between two token arrivals;

(b) it is a worst-case scenario that, simultaneously with condition 1(a), the speci"c message will be the last in a queue with a length of $n s k$;
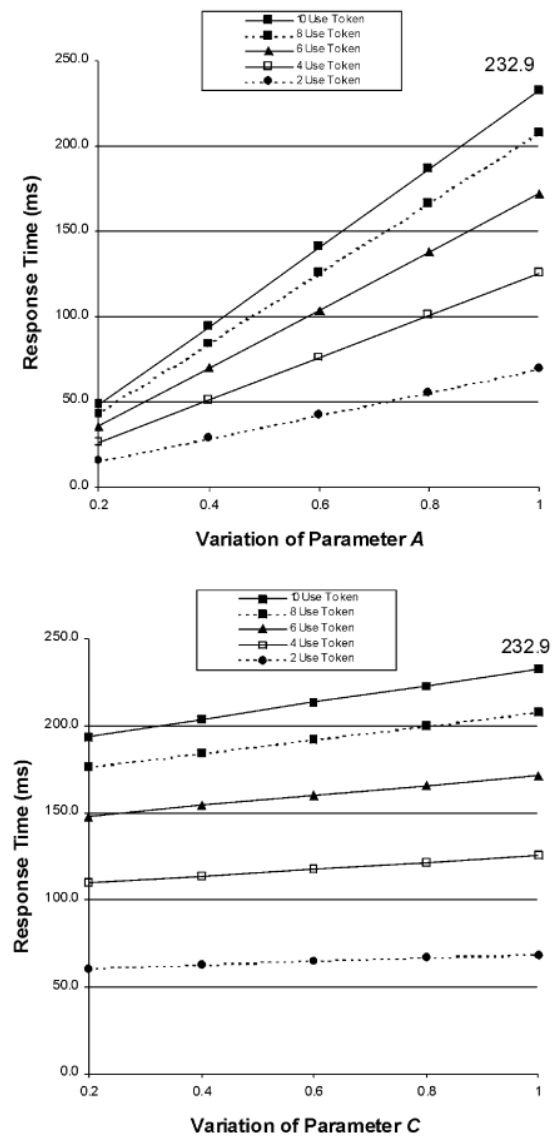

(c) it is a worst-case scenario that, simultaneously with conditions 1 (a) and 1(b), multiple request for message streams will occur just after a token visit, thus imposing an additional token rotation in the overall access time;

(2) In the virtual token cycle factor $(V)$ :

(a) it is a worst-case scenario all master stations will use the token for sending a message;

(b) it is a worst-case scenario that, simultaneously with condition 2(a), all stations will transfer their longest message cycle;

(c) it is a worst-case scenario that the time $7 \times \mathbf{x b p}$ is the master's reaction time;

(d) and "nally, it is a worst-case scenario that the time $30 \times \mathbf{x} p$ is the slave's turnaround time.

Assume a scenario of a P-NET network with ten masters, where each master supports ten message streams. If all masters have a maximum message cycle length of $100 \times b p$ (request and response frame lengths, but, for this analysis without the slave's turnaround time), then the worst-case response time for a message
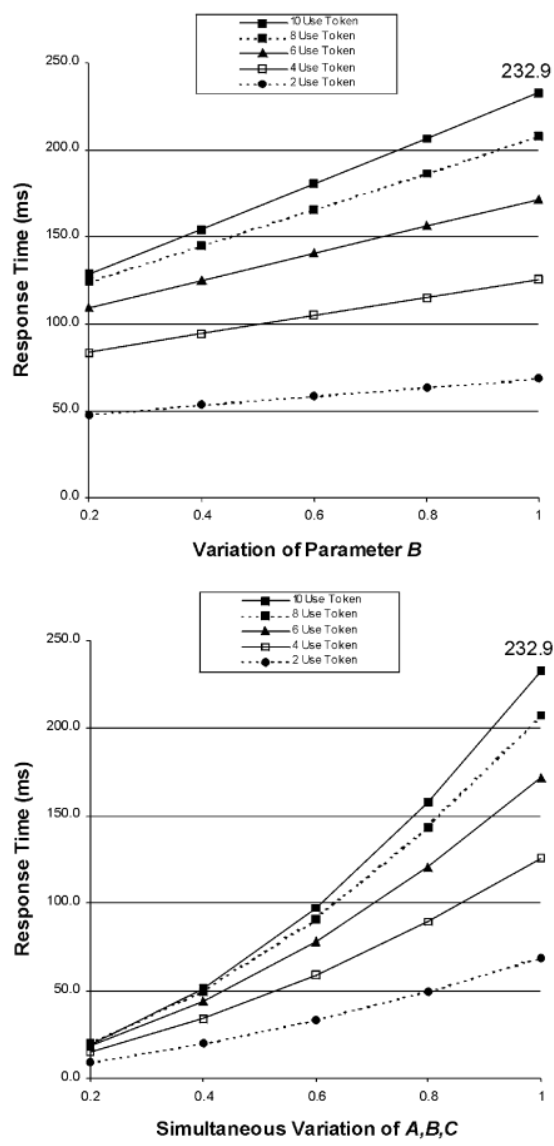

Fig. 8. Level of pessimism on the evaluation of the upper bound of the response time for single-segment message streams. 

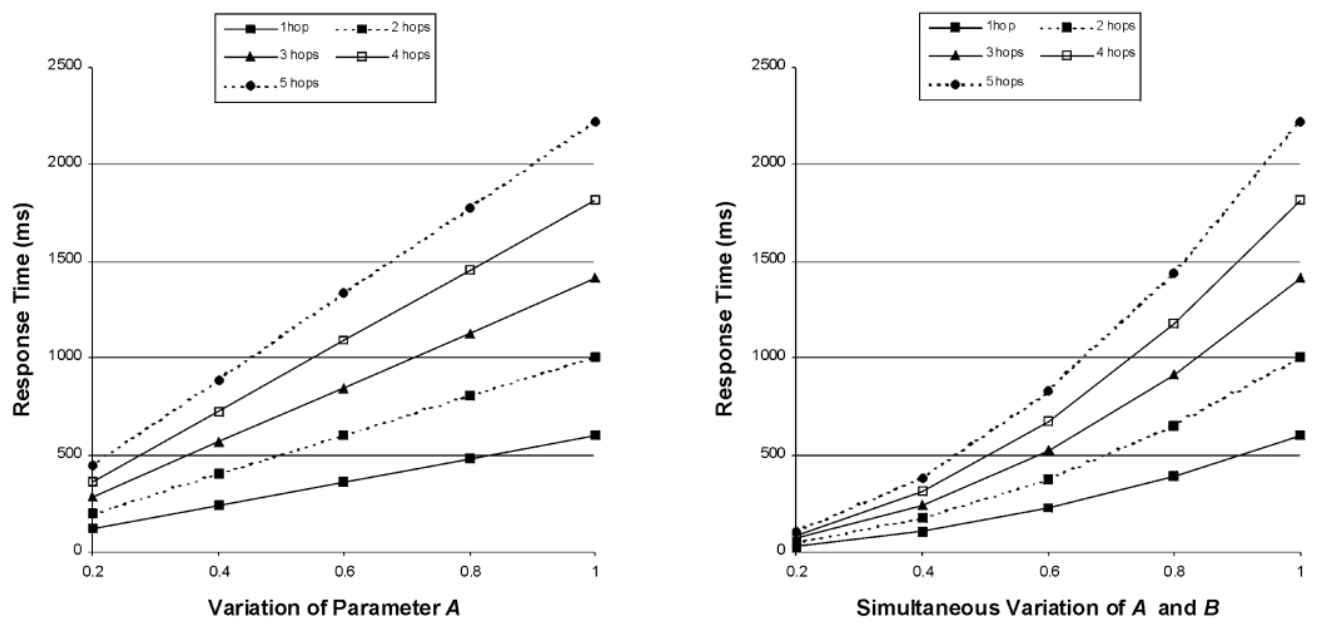

Fig. 9. Level of pessimism on the evaluation of the upper bound of the response time for multi-hop message streams.

cycle in that station is (again ignoring $2 k$ $V=\sum_{i-1, \ldots, 10}(7 \times b p+130 \times b p+40 \times b p)=17700 \times$ $b p=23.1 \mathrm{~ms}$, with a slave turnaround time equal to $30 \times b p$. Therefore, the upper bound for the response time of any message request in any master in the network will be : $R=10 \times V+137 \times b p=232.9 \mathrm{~ms}$.

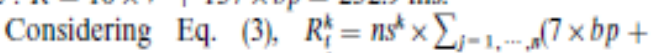
$\left.C_{\max }+40 \times b p\right)+7 \times b p+C_{i}^{k}$, the following equation:

$$
\begin{aligned}
R_{i}^{k}= & \mathbf{A} \times n s^{k} \times\left[(n-\mathbf{D}) \times\left(\mathbf{B} \times C_{\max }^{*}+40 \times b p+\mathbf{C}\right.\right. \\
& \times 37 \times b p)+\mathbf{D} \times 10 \times b p]+C_{\max }^{*}+\mathbf{C} \times 37
\end{aligned}
$$

can be used to access the impact of each of the factors identified as contributors to the overall pessimism level.

A represents the aggregate probability of the simultaneous occurrence of situations 1(a), 1(b) and 1(c). B represents the probability of a reduction in the average length of messages cycles in a complete token rotation. $\mathrm{C}$ represents the probability of a reduction in the average time for both the master's reaction and the slave's turnaround times. Finally, $\mathbf{D}$ represents the average number of masters not using the token in a complete token rotation. The term $\mathbf{D} \times 10 \times b p$ is included because it represents the token-passing time for masters not using the token (refer to Fig. 2 for clarification). $C_{\max }^{*}$ denotes the message length excluding the slave's turnaround time $\left(C_{\max }=C_{\max }^{*}+30 \times b p\right)$.

Assuming the above scenario (ten masters, ten message streams in each, etc.), the Eq. (9) can be re-written as

$$
\begin{aligned}
R=\{ & \mathbf{A} \times 10 \times[(10-\mathbf{D}) \times(\mathbf{B} \times 100+40+\mathbf{C} \times 37) \\
& +\mathbf{D} \times 10]+100+\mathbf{C} \times 37\} / 76.8(\mathrm{~ms}) .
\end{aligned}
$$

Fig. 8 illustrates the impact of each parameter on the evaluation of the upper bound of the response time. When all parameter values are set to 0.6 , the upper bound for the response time is $58.6 \mathrm{~ms}$, which compared to $232.9 \mathrm{~ms}$, gives a reduction of about $25 \%$.
In the case of the response times of multi-hop message streams (Eqs. (7) and (8)), the pessimism level is obviously higher, and increases as the number of intermediate hopping devices increases.

A simplified version of $\mathrm{Eq}$. (8) can be used, as $n s^{1}=n s^{2}=\cdots=n s^{10}$ (again, parameters $\phi$ and $\lambda$ are ignored):

$R=(2 \times h+1) \times n s^{k} \times\left(V+7 \times b p+C^{n}\right)$

and the following equation can be used to access the level of pessimism:

$R=(2 \times h+1) \times \mathbf{A} \times n s^{k} \times\left(\mathbf{B} \times V+7 \times b p+C^{2}\right)$.

In Eq. (12), $h$ represents the number of hopping devices a message stream is relayed through. Parameter $\mathrm{A}$ is a reducing factor related to the number of streams in each outgoing queue. Parameter $\mathbf{B}$ is a reducing factor for the average token-rotation time.

Assuming that all segments have ten masters, each with ten message streams, and with $100 \times b p$ as the maximum length of the message cycles, then in each segment $V=10 \times(7 \times b p+100 \times b p+40 \times b p)=19.1 \mathrm{~ms}$. If $C^{\prime}$ is assumed to be, on average, $80 \times b p$, then, $R=(2 \times h+1) \times A \times 10 \times(B \times 19.1+1.1) \mathrm{ms}$.

Fig. 9 illustrates the level of pessimism as a function of the number of hopping devices a message stream is relayed through. It is easy to see, from the equation $R=(2 \times h+1) \times A \times 10 \times(B \times 19.1+1.1) \mathrm{ms}$ that the individual contributions of $\mathbf{A}$ and $\mathbf{B}$ are similar. Therefore, only trends for parameter $\mathbf{A}$ and aggregation of $\mathbf{A}$ and B are shown in the graphs.

\section{References}

Afnor. (1990). Normes FIP NF C46-601 to NF C46-607. Union Technique de l'ElectriciteH . 
Cardoso, A., \& Tovar, E. (1996). Industrial communication networks: issues on heterogeneity and internetworking. Proceedings of the sixth international conference on yexible automation and intelligent manufacturing (FAIM'96), Atlanta, USA (pp. 139\}148).

Cenelec. (1996). General purpose "eld communication system. EN 50170, vol. 1/3 (P-NET), Vol. 2/3 (PROFIBUS), vol. 3/3 (FIP), Cenelec.

Jenkins, C. (1997). P-NET as a European Fieldbus Standard EN 50170, Vol. 1. Institute of Measurement and Control Journal, 30, April, 75$\} 79$.

Lenhart, G. (1993). A "eldbus approach to local control networks. Advances in Instrumentation and Control, 48(1), 357\}365.

Pnet. (1994). The P-NET Standard. International P-NET User Organisation ApS.
Pro"bus. (1992). PROFIBUS Standard DIN 19245 part I and II. Translated from German, Pro"bus Nutzerorganisation e.V.

Tindell, K., Burns, A., \& Wellings, A. (1995). Analysis of hard real-time communications. Journal of Real-Time Systems, 9, 147\}171.

Tovar, E., \& Vasques, F. (1998a). Scheduling real-time communications with P-NET. Digest of the IEE Real-Time Systems Colloquium, York, England, Digest No. 98/306, (pp. 9/1\}9/5).

Tovar, E., \& Vasques, F. (1998b). Pre-run-time schedulability analysis of P-NET "eldbus networks. Proceedings of the 24th annual conference of IEEE industrial electronics society (IECON'98), vol. 1/4 (pp. 236\}241). Germany: Aachen. 